\title{
SUPERVIVENCIA EN PACIENTES CON TUBERCULOSIS INFECTADOS POR VIH. ESTUDIO DE LOS FALLECIMIENTOS EN LOS PRIMEROS NUEVE MESES DE TRATAMIENTO
}

\author{
Meritxell Falqués Casanovas (1), Klaus Langohr (2), Guadalupe Gómez Melis (1), Patricia García \\ de Olalla Rizo (3), Josep M. Jansà López del Vallado (3) y Joan A. Caylà Buqueras (3). \\ (1) Departament d'Estadística, Universitat Politècnica de Catalunya. Barcelona. \\ (2) Departamento de Estadística. Universidad de Dortmund. Alemania. \\ (3) Servicio de Epidemiología. Institut Municipal de la Salut, Barcelona, Unidad de Investigación en Tuberculosis \\ en Barcelona.
}

\section{RESUMEN}

Fundamento: En las personas con luberculusis infectadas por el VIH, se ha observado una elevada letalidad durante las primeras semanas desde el inicio de la terapia antituberculosa. En el presente estudio se analiza la supervivencia de las mismas en el marco de un cstudio de cohortes retrospectivo.

Métodos: Se incluyeron 1.135 individuos que correspondían a aquellos por el VIH mayores de quince años y residentes en Barcelona, registrados por el Programa de Prevención y Control de la Tuberculosis de Barcelona y que fueron diagnosticados entre 1988 y 1993. Las variables analizadas fueron edad, sexo, antecedentes de prisión, distrito municipal, grupo de riesgo, porcentaje de linfocitos $\mathrm{T} \mathrm{CD} 4+$, prueba de la tuberculina. diagnóstico de sida (según criterio ('DC-1987), patrón radiológico, bacteriología y localización anatómica de la tuberculosis. Se utilizó el método semiparamétrico de Cox, las curvas de Kaplan-Meier y la prueba de log-rank.

Resultados: La probabilidad de supervivencia a los nueve meses fue del $77 \%$, con amplias variaciones en los diversos subgrupos. Las únicas variables significativas en el modelo multivariado de Cox fueron sida, porcentaje de linfocitos $T$ $\mathrm{CD} 4+\mathrm{y}$ su interacción. El riesgo de morir para un individuo $\sin$ SIDA y porcentaje de linfocitos T CD4 + inferior o igual al $14 \%$ fue 7.69 veces mayor que el riesgo de morir para un individuo sin SIDA y porcentaje de linfocitos T CD4 - superior al 14\%.

Conclusiones: La supervivencia en las personas con tuberculosis infectadas por VIII es muy variable. $L$ as que fallecieron a corto plazo fueron las diagnosticadas de sida al iniciar el tratamiento antituberculoso y que, además, tenian un porcentaje de linfocitos T CD4+ inferior o igual al 14\%

Palabras clave: CD4. Prueba de la tubcrculina. Sida. Supervivencia. Tuberculosis.

Correspondencia:

Meritxell Falqués Casanovas.

C Cardenal Reig, 18. Esc $\Lambda 6 .^{\circ} 4 .^{\circ}$

08028 Barcelona

\section{ABSTRACT}

\section{Survival in Patients with Tuberculosis Infected with HIV. Study of the Deaths within the First Nine Months}

Background: Among the individuals with tuberculosis who are infected with HIV a high degree of lethality has been found to exist during the first few weeks following the start of tuberculosis treatment. In this study, the survival of these individuals is studied within the framework of a retrospective cohort study.

Methods: This study included 1135 subjects infected by HIV over age fifteen and residents of Barcelona who were registered by the Barcelona Tuberculosis Prevention and Control Program and were diagnoses within the $1988-1993$ period. The variables analyzed were age, gender, former imprisonment, municipal district, risk group, percentage of $\mathrm{T} \mathrm{CD} 4+$ lymphocytes, tuberculin test, AIDS diagnosis (as per CDC-1987), X-ray pattern, bacteriology and part of the body affected by the ruberculosis. The Cox semiparametric method, the Kaplan-Meir curves and the log-rank test were employed.

Results: A $77 \%$ probability of survival during the first nine months was found to exist, with wide-ranging variations among the different subgroups. The only significant variables in the Cos multivariate model were AJDS, the percentage of $\mathrm{T}$ $\mathrm{CD} 4+$ lymphocytes and their interaction. The risk of death for an individual not having AIDS and a T CD4+ lymphocyte percentage of $14 \%$ or lower was 7.69 times higher than the risk of dying for an individual not having AIDS who had a T CD4+ lymphocyte percentage of above $14 \%$.

Conclusions: The survival of those individuals having tuberculosis who are infected with HIV varies greatly. Those who died in the short term were diagnosed as having AIDS on starting the tuberculosis treatment and who also had a T CD4+ lymphocyte percentage of $14 \%$ or lower.

Key words: CD4. Tuberculin test. AIDS. Survival. Tuberculosis. 


\section{INTRODUCCIÓN}

En el ámbito mundial, desde el inicio de la epidemia de sida, más de 14 millones de personas han muerto a causa de la infección $\mathrm{VIH}^{1}$. En los Estados Unidos y Europa occidental las tasas de mortalidad entre los pacientes infectados por el VIH han disminuido drásticamente desde septiembre de $1995^{2,3}$, atribuyéndose una parte importante de esta reducción a los nuevos tratamientos antirretrovirales ${ }^{4}$. Además, otras medidas, como son las mejoras en los tratamientos de las profilaxis de las infecciones oportunistas y cl mejor acceso a los servicios de salud, contribuyen a mejorar la supervivencia de estos pacientes ${ }^{5}$. A pesar de estas mejoras en el ámbito mundial, el número de pacientes con sida sigue creciendo y lo mismo sucede con la mortalidad que ocasiona, influyendo en todo ello la falta de tratamientos y la deficiente infraestructura de los servicios de salud en muchos países?

En España, no se objetivaron declives significativos de la mortalidad global debida al sida, hasta que no se introdujeron los nuevos tratamientos antirretrovirales en el tratamiento de la infección VIH. Así, en Barcelona la probabilidad de supervivencia por sida al año y medio, entre 1987 y 1993 , se situaba entre el $53 \%$ y el $57 \%$, apreciándose que los más jóvenes, aquellos sujetos cuyo modo de transmisión de la infección no era el uso de droga por vía parenteral (UDVP) y aquellos en que la tuberculosis (TBC) fue la enfermedad diagnóstica de sida, eran factores predictores de mejor supervivencia ${ }^{6}$.

En los enfermos tuberculosos infectados por el VIH, se ha observado una letalidad relativamente alta, durante los primeros días y las primeras semanas, desde el inicio de la terapia antituberculosa, fenómeno que no ha sido analizado con detenimiento hasta el momento actual ${ }^{7}$. En este sentido, el objetivo del estudio es determinar la supervivencia y sus factores predictores en los nueve primeros meses tras el inicio del tratamiento antituberculoso en perssonas infectadas por el VIII.

\section{SUJETOS Y MÉTODOS}

Se incluyeron todos los pacientes tuberculosos, mayores de quince años, infectados por el VIH, registrados por el Programa de Prevención y Control de la Tuberculosis de Barcelona, que correspondían a personas residentes en la ciudad y que iniciaron tratamiento entre el 1 de enero de 1988 y el 31 de diciembre de 1993. La fecha de cierre del estudio fue el 30 de septiembre de 1995. En los sujetos que presentaron varios episodios sólo se consideró el primer diagnóstico de $\mathrm{TBC}$.

El Sistema de Vigilancia Epidemiológica Activa (SVEA), del Servicio de Epidemiología del Institut Municipal de la Salut (IMS), tiene como objetivo recoger tanto los casos declarados por los médicos (a través del sistema de enfermedades de declaración obligatoria, EDO) como los no notificados. Se controlan los resultados microbiológicos correspondientes a micobacterias, las altas hospitalarias, el registro de mortalidad y se hace un contraste sistemático entre los registros de TBC y de sida, también incluido en el SVEA. Ambos registros son actualizados periódicamente $\mathrm{y}$, de este modo, se consigue que dichos registros alcancen un grado de exhaustividad muy elevado.

A cada uno de los sujetos registrados se le realizó una encuesta que recoge variables de interés clínico y epidemiológico. Las variables que se han tenido en cuenta en este estudio son las siguientes: 1) variables sociodemográficas: edad, sexo, antecedentes penitenciarios, distrito municipal de residencia, modo de transmisión del VIH; 2) variables clínicas: porcentaje de linfocitos T CD4+ (recogido como máximo dos meses antes o después del diagnóstico de la TBC), prueba de la tuberculina (realizada en el momento del diagnóstico), diagnóstico de sida, tipo de tratamiento, patrón radiológico, bac- 
teriología, y localización anatómica de la TBC (pulmonar exclusivamente, extrapulmonar exclusivamente y mixta, que comprende la afectación de dos o más órganos y la forma miliar o diseminada); 3) variables temporales: fecha de inicio del tratamiento antituberculoso y fecha de fallecimiento o de censura.

\section{Definiciones}

Caso de tuberculosis: aquella persona a la que se le prescribe quimioterapia antituberculosa y ésta se mantiene hasta el momento previsto de la finalización, a menos que muera o presente cfectos secundarios importantes. Infección por el VIH: se determina si el resultado de la prueba ELISA (enzime-linked immunosorbent assay) es positivo en el momento del diagnóstico de la TBC y es confirmado mediante un Western blot.

Sida: se consideró que una persona tenía sida, si en el momento del diagnóstico de la TBC, presentaba una de las enfermedades diagnósticas de sida incluidas en la definición de los CDC de agosto de $1987^{8}$.

Prueba de la tuberculina: Dicha prueba se realizó de acuerdo con la técnica de Mantoux, aplicando 2 TU de PPD RT-23 intradérmica, la lectura se realizó entre las 48 y las 72 horas posteriores. Induraciones mayores o iguales a 5 fueron consideradas positivas 9 .

Tiempo de supervivencia: Número de días transcurridos desde el inicio del tratamiento antituberculoso hasta la fecha de fallecimiento. O bien hasta el 30 de septiembre de 1995 para aquellos individuos que permanecían vivos en dicha fecha. Consecuentemente, aquellas personas vivas cuando el estudio concluyó, se consideran censuradas por la derecha, porque la muerte no ha sido observada. La misma consideración se aplicó a los que cambiaron de residencia o fueron perdidos durante el estudio aunque, en estos casos, la fecha de censura fue la del último control y no el 30 de septiembre de 1995.

Supervivencia a corto plazo: Período transcurrido entre el inicio del tratamiento y el fallecimiento del paciente o su pérdida, si ésta acon- tece en los primeros nueve meses. Se considera este período de tiempo, ya que el tratamiento de nueve meses es una pauta estándar para pacientes tuberculosos infectados por el $\mathrm{VIH}^{9}$.

La tasa de incidencia media anual se define como el número de casos multiplicado por 100.000 , dividido por la población según el padrón del 91 y dividido por 6 (número de años del estudio).

\section{Análisis estadístico}

En el estudio se incluye un análisis descriptivo univariado y bivariado de las variables. Se ha utilizado la prueba $\chi^{2}$ para estudiar relaciones entre variables categóricas y la prueba de la $t$ de Student para variables continuas normales. Para la estimación de la función de supervivencia se utilizó el método Kaplan-Meier, y para comparar curvas de supervivencia entre distintas categorías de una misma variable se aplicó la prueba del Log-rank. Finalmente, se ajustó un modelo multivariado de riesgos proporcionales de Cox a aquellos pacientes cuyo nivel de linfocitos $T$ $\mathrm{CD} 4+$ ha sido informado y para aquellas variables que cumplen la condición de riesgos proporcionales y que son significativas $(\mathrm{p}<0.05)$ a nivel univariado. Los modelos se validaron utilizando el análisis de residuos de Cox-Snell ${ }^{10}$. Los datos se procesaron con dBASE-IV y se analizaron con los paquetes estadísticos SPSS-Win ${ }^{11}$ y EGRET ${ }^{12}$.

\section{RESULTADOS}

\section{Características descriptivas de la población de estudio (tabla 1)}

Entre las 1.135 personas incluidas en el estudio, destacaba que la medid de edad era de 32,44 años con una desviación estándar (DE) de 8,8; dicha pobla- 
ción era mayoritariamente masculina $(82,6 \%)$, usuaria de drogas por vía parenteral (UDVP) $(73,4 \%)$ y había sido diagnosticada de sida en el momento del diagnóstico de la TBC $(68,7 \%)$. Ciutat Vella, el distrito más deprimido socioeconómicamente de la ciudad $^{13}$, destacaba como el más afectado, con una tasa de incidencia media anual de
60,8 casos por cada 100,000 habitantes, seguida de Nou Barris, con una incidencia media anual igual a 17 .

Observando la evolución de los casos entre 1988 y 1993 , se detectaron diferencias significativas en la distribución de los mismos según edad $(\mathrm{p}<0,001)$, modo de trans-

Tabla 1

Características descriptivas de los pacientes tuberculosos infectados por VIH. Barcelona (1988-1993)

\begin{tabular}{|c|c|c|c|}
\hline Variable & Categoria & Número & Porcentaje \\
\hline \multirow[t]{2}{*}{ Sexo } & Hombres & 938 & 82.6 \\
\hline & Mujeres & 197 & 17.4 \\
\hline \multirow[t]{2}{*}{ Antecedentes de prisión } & Sí & 273 & 24.1 \\
\hline & No & 862 & 75.9 \\
\hline \multirow[t]{6}{*}{ Grupo de riesgo } & UDVP* & 833 & 73.9 \\
\hline & Homosexuales & 156 & 13.7 \\
\hline & Hemofilicos/Hemotransfundidos & 10 & 0.9 \\
\hline & Heterosexuales & 41 & 3.6 \\
\hline & UDVP + Homosexuales & 29 & 2.6 \\
\hline & Desconocido & 66 & 5.8 \\
\hline \multirow[t]{3}{*}{ Porcentaje CD4 } & Hasta $14 \%$ & 288 & 25.4 \\
\hline & Más de $14 \%$ & 239 & 21.1 \\
\hline & Desconocido & 608 & 53.6 \\
\hline \multirow[t]{3}{*}{ Prueba tuberculina } & Positiva & 245 & 21.6 \\
\hline & Negativa & 325 & 28.6 \\
\hline & Desconocida & 565 & 49.8 \\
\hline \multirow[t]{2}{*}{ Sida (CDC-1987) } & Si & 780 & 68.7 \\
\hline & No & 355 & 31.3 \\
\hline \multirow[t]{4}{*}{ Radiología } & Normal & 181 & 15.9 \\
\hline & Anormal cavitaria & 160 & 14.1 \\
\hline & Anormal no cavitaria & 740 & 65.2 \\
\hline & No practicada & 54 & 4.8 \\
\hline \multirow[t]{5}{*}{ Bacteriología } & Microscopía positiva & 127 & 37.6 \\
\hline & Sólo cultivo positivo & 361 & 31.8 \\
\hline & Negativo & 209 & 18.4 \\
\hline & No determinada & 138 & 12.2 \\
\hline & Otros** & 38 & 3.3 \\
\hline \multirow[t]{4}{*}{ Localización TBC } & Pulmonar & 529 & 46.6 \\
\hline & Extrapulmonar & 393 & 34.6 \\
\hline & Mixta & 201 & 17.7 \\
\hline & Desconocida & 12 & 1.1 \\
\hline
\end{tabular}

* UDVP: Usuarios de drogas por vía intravenosa.

** La mayoría corresponden a pacientes diagnosticados por critcrios clínico-radiológicos o por la prueba ADA. 
misión ( $\mathrm{p}<0,001)$, nivel de CD4 $(\mathrm{p}<0,001)$ y la prueba de tuberculina $(\mathrm{p}<0,001)$. Para el total de la ciudad, en 1988 se observan 155 casos nuevos (tasa de incidencia de 12 casos por cada 100.000 habitantes de edades comprendidas entre 15 y 74 años) y, a partir de 1990, se producen del orden de 200 casos cada año, con un máximo de 217 en 1991 , dicha tasa es de 16,9/100.000. Mientras que la media de edad aumentó cada año, pasando de 30,4 años (DE: 3,0) en 1988 a 34,7 años (DE: 4,0), el porcentaje de pacientes UDVP descendió ligeramente cada año, desde $1988(81,2 \%)$ hasta $1993(64,6 \%)$; el de los hombres con prácticas homosexuales (HMS) ascendió de 11\% a 18,7\% durante el mismo período y la tendencia del colectivo de personas con prácticas heterosexuales de riesgo (HTS) aumentó, desde $0 \%$ en 1988 hasta alcanzar un $11 \%$ en 1993 . El porcentaje de pacientes con un nivel de linfocitos $T$ CD4 inferior o igual al $14 \%$ aumentó de $10,7 \%$ en 1988 a $60,0 \%$ en 1993 . También se observó que el porcentaje de pacientes tuberculín negativos ascendió a lo largo de este período, pasando de un $40 \%$ en 1988 a un $60 \%$ en 1993.

\section{Estudio descriptivo de los fallecimientos a corto plazo}

Este análisis se basó en 1.033 sujetos. Se incluyeron los fallecidos durante los nueve primeros meses y los que sobrevivieron más de 9 meses. De un total de 247 (23\%) fallecimientos en los primeros 9 meses, 38 (15\%) se produjeron en los primeros 10 días y $93(38 \%)$ en los primeros 50 días. La letalidad en los primeros 9 meses ascendió, de un $17.3 \%$ en 1988 a un $25 \%$ en 1993 , aunque esta tendencia no resulta significativa.

Al comparar las características de las personas que fallecicron durantc los primeros 9 meses con los que viven más de nueve, las primeras se caracterizan por ser de mayor edad, por no presentar antecedentes de prisión, ser mayoritariamente HMS, tener un nivel de $\mathrm{CD} 4</=14 \%$, tener un resultado negativo en la prueba de la tuberculina y estar diagnosticados de sida (tabla 2). Se observó también que la prueba de la tuberculina fue negativa en el $77,4 \%$ de los fallecidos a corto plazo, mientras que, cntrc los pacientes que sobrevivieron más de 9 meses, el resultado de la prueba resultó negativo en el 53,5\% ( $p<0.010$, sin tener en cuenta los valores desconocidos). El 87\% de pacientes del grupo con supervivencia menor a nueve meses fueron casos de sida cuando empiezan el tratamiento antituberculoso. Sin embargo, en el grupo de largo plazo esta cifra se reduce al $63,4 \%$ $(\mathrm{p}<0.001)$.

\section{Análisis de Supervivencia}

Destacó que la supervivencia de los pacientes tuberculín positivos fue significativamente superior a la de los tuberculín negativos y a la de los que no tienen la prueba de la tuberculina informada, $\mathrm{p}<0.001$ (figura 1). Asimismo, la supervivencia de los pacientes con CD4>14\% fue superior a la supervivencia de los que presentan un nivel de CD4 $\angle /=14 \%$ o a la de los no observados $(\mathrm{p}<0.001)$ (figura 2). Además de las variables anteriormente citadas, el no haber sido diagnosticado de sida, el patrón radiológico anormal cavitario y la TBC exclusivamente tuberculosis pulmonar, presentaron mejor supervivencia (tabla 3 ).

Se observó también que la supervivencia de aquellos pacientes con prueba de la tuberculina no informada se comportaba de forma similar a la supervivencia de los tuberculín negativos, $\mathrm{p}=0,52$ (figura 1 ), lo que hace sospechar que los valores no observados de la prueba de la tuberculina no están distribuidos aleatoriamente. Por otro lado, tal como se desprende de la tabla 3, se observan diferencias significativas entre la supervivencia de los pacientes tuberculín positivos respecto de los que tienen la prueba negativa. 
Tabla 2

Comparación de las caracteristicas de los pacientes tuberculosos infectados por VIH según fallecieron a corto plazo $(\leq 9$ meses) o vivieran más de 9 meses. Barcelona (1988-1993)

\begin{tabular}{|c|c|c|c|c|c|c|}
\hline \multirow{2}{*}{$\begin{array}{l}\text { Variable } \\
\text { Edad (años) }\end{array}$} & \multirow{2}{*}{ Categoría } & \multicolumn{2}{|c|}{$\leq 9$ meses } & \multicolumn{2}{|c|}{$>9$ meses } & \multirow{2}{*}{$\begin{array}{l}\text { Valor } p \\
<0.001\end{array}$} \\
\hline & & $X=36.0(N)$ & $\begin{array}{c}D E . *=10.84 \\
(\%(1))\end{array}$ & $X=31.5(\mathrm{~N})$ & $\begin{array}{c}\text { d.e. }{ }^{*}=7.57 \\
(\%)\end{array}$ & \\
\hline Sexo & $\begin{array}{l}\text { Hombres } \\
\text { Mujeres }\end{array}$ & $\begin{array}{r}204 \\
43\end{array}$ & $\begin{array}{l}82.6 \\
17.4\end{array}$ & $\begin{array}{l}650 \\
136\end{array}$ & $\begin{array}{l}82.7 \\
17.1\end{array}$ & 0.963 \\
\hline $\begin{array}{l}\text { Antecedentes } \\
\text { prisión }\end{array}$ & $\begin{array}{l}\text { Sí } \\
\text { No }\end{array}$ & $\begin{array}{r}27 \\
220\end{array}$ & $\begin{array}{l}10.9 \\
89.1\end{array}$ & $\begin{array}{l}213 \\
573\end{array}$ & $\begin{array}{l}27.1 \\
72.9\end{array}$ & $<0.001$ \\
\hline Distrito municipal & $\begin{array}{l}\text { Ciutat Vella } \\
\text { Resto } \\
\text { Desconocido }\end{array}$ & $\begin{array}{r}55 \\
191 \\
1\end{array}$ & $\begin{array}{r}22.3 \\
77.3 \\
0.4\end{array}$ & $\begin{array}{l}179 \\
607 \\
-\end{array}$ & $\begin{array}{l}22.8 \\
77.2 \\
-\end{array}$ & 0.855 \\
\hline Grupo de riesgo & $\begin{array}{l}\text { UDVP } \\
\text { HMS } \\
\text { HTS } \\
\text { Desconocido }\end{array}$ & $\begin{array}{r}154 \\
43 \\
13 \\
37\end{array}$ & $\begin{array}{r}62.3 \\
17.4 \\
5.3 \\
15.0\end{array}$ & $\begin{array}{r}615 \\
91 \\
24 \\
56\end{array}$ & $\begin{array}{r}78.2 \\
11.6 \\
3.1 \\
7.1\end{array}$ & 0.001 \\
\hline Porcentaje CD4 & $\begin{array}{l}\text { Hasta } 14 \% \\
\text { Más del } 14 \% \\
\text { Desconocido }\end{array}$ & $\begin{array}{r}82 \\
36 \\
129\end{array}$ & $\begin{array}{l}33.2 \\
14.6 \\
52.2\end{array}$ & $\begin{array}{l}182 \\
186 \\
418\end{array}$ & $\begin{array}{l}23.2 \\
23.7 \\
53.2\end{array}$ & $<0.001$ \\
\hline Prueba tuberculina & $\begin{array}{l}\text { Positivo } \\
\text { Negativo } \\
\text { Desconocido }\end{array}$ & $\begin{array}{r}21 \\
72 \\
154\end{array}$ & $\begin{array}{r}8.5 \\
29.1 \\
62.3\end{array}$ & $\begin{array}{l}198 \\
228 \\
360\end{array}$ & $\begin{array}{l}25.2 \\
29.0 \\
45.8\end{array}$ & $<0.001$ \\
\hline Sida & $\begin{array}{l}\text { Sí } \\
N_{0}\end{array}$ & $\begin{array}{r}215 \\
32\end{array}$ & $\begin{array}{l}87.0 \\
13.0\end{array}$ & $\begin{array}{l}498 \\
288\end{array}$ & $\begin{array}{l}63.4 \\
36.6\end{array}$ & $<0.001$ \\
\hline Radiologia & $\begin{array}{l}\text { Normal } \\
\text { Patrón cavitario } \\
\text { Patrón no cavitario } \\
\text { Desconocida }\end{array}$ & $\begin{array}{r}37 \\
27 \\
174 \\
9\end{array}$ & $\begin{array}{l}15.5 \\
11.3 \\
73.1\end{array}$ & $\begin{array}{r}128 \\
116 \\
503 \\
39\end{array}$ & $\begin{array}{r}16.3 \\
14.7 \\
64.0 \\
5.0\end{array}$ & 0.190 \\
\hline Bacteriologia & $\begin{array}{l}\text { Positivo por } \\
\text { micros. } \\
\text { Cultivo positivo } \\
\text { Negativo } \\
\text { Desconocida }\end{array}$ & $\begin{array}{r}44 \\
127 \\
41 \\
35\end{array}$ & $\begin{array}{l}17.8 \\
51.4 \\
16.6 \\
14.2\end{array}$ & $\begin{array}{r}125 \\
432 \\
141 \\
88\end{array}$ & $\begin{array}{l}15.9 \\
55.0 \\
17.9 \\
11.2\end{array}$ & 0.645 \\
\hline Localización TBC & $\begin{array}{l}\text { Pulmonar } \\
\text { Extrapulmonar } \\
\text { Mixta } \\
\text { Desconocida }\end{array}$ & $\begin{array}{r}122 \\
81 \\
39 \\
5\end{array}$ & $\begin{array}{r}49.4 \\
32.8 \\
15.8 \\
2.0\end{array}$ & $\begin{array}{r}367 \\
273 \\
142 \\
4\end{array}$ & $\begin{array}{r}46.7 \\
34.7 \\
18.1 \\
0.5\end{array}$ & 0.602 \\
\hline Inicio tratamiento & $\begin{array}{l}1988 \\
1989 \\
1990 \\
1991 \\
1992 \\
1993\end{array}$ & $\begin{array}{l}26 \\
35 \\
40 \\
53 \\
44 \\
49\end{array}$ & $\begin{array}{l}10.5 \\
14.2 \\
16.2 \\
21.5 \\
17.8 \\
19.8\end{array}$ & $\begin{array}{l}119 \\
113 \\
142 \\
142 \\
134 \\
136\end{array}$ & $\begin{array}{l}15.1 \\
14.4 \\
18.1 \\
18.1 \\
17.0 \\
17.3\end{array}$ & 0.402 \\
\hline Total & & $247 / 1033$ & 23.9 & $786 / 1033$ & 76.1 & \\
\hline
\end{tabular}

* $\mathrm{DE}=$ Desviación estándar.

UDVP: Usuario de drogas por vía parenteral.

HMS: Hombres con practicas homosexuales.

HTS: Prácticas heterosexuales de riesgo.

' Ciutat Vella: Distrito más deprimido socio-económicamente de la ciudad. 
Figura 1

Función de supervivencia para la variable «Prueba de la tuberculina»

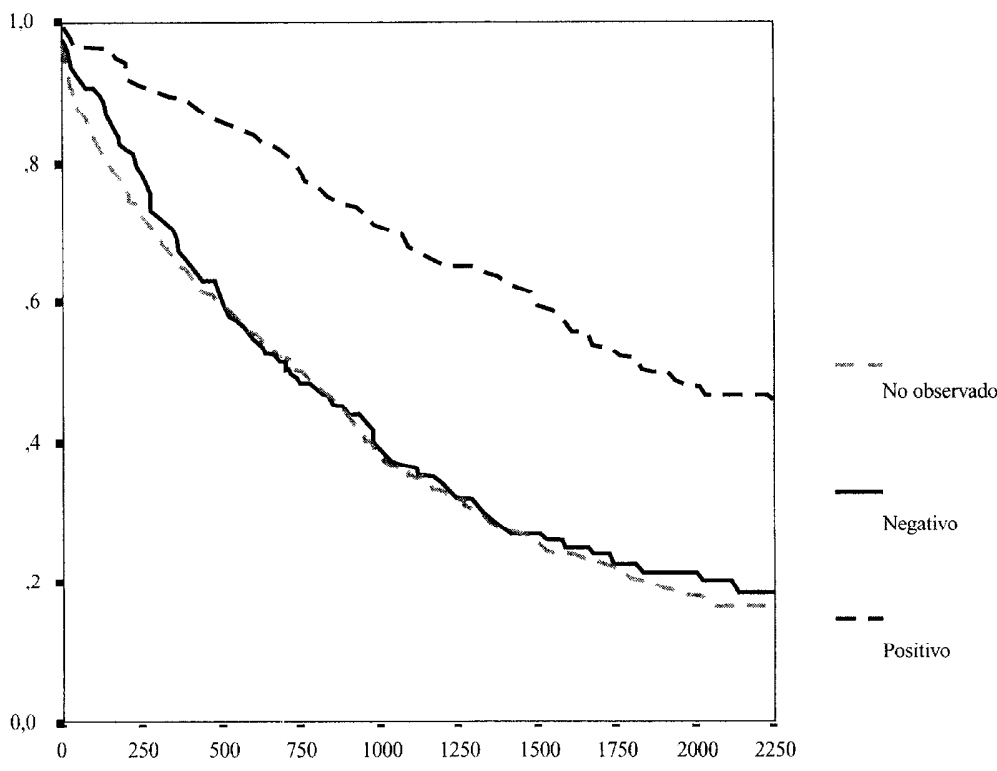

Días

Figura 2

Función de supervivencia para la variable «Linfocitos T CD4+»

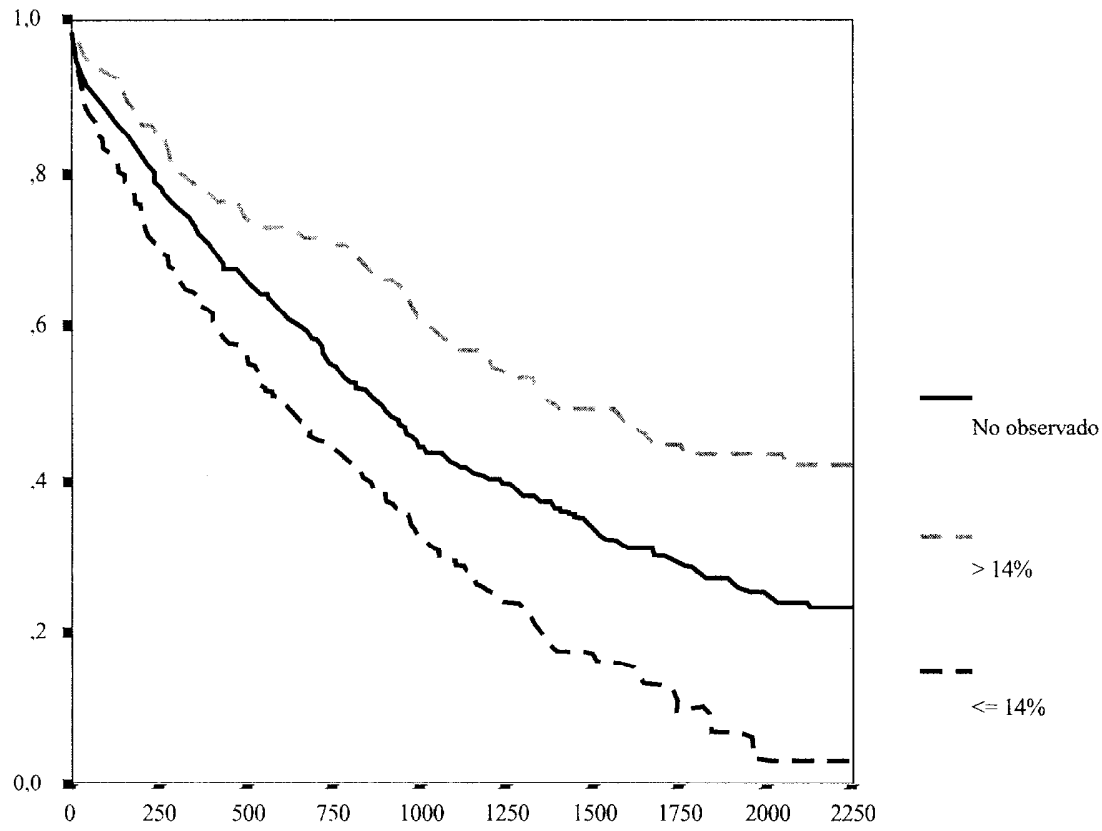

Días 
Tabla 3

Caracterización de la supervivencia según las categorías de cada variable de los pacientes tuberculosos infectados por VIH. Barcelona (1988-1993)

\begin{tabular}{|c|c|c|c|c|c|}
\hline Variable & Categoria & $N$ & $\%$ fallecidos & Mediana* & Valor $p$ \\
\hline \multirow[t]{2}{*}{ Sexo } & Hombres & 876 & 65.53 & 898 & 0.998 \\
\hline & Mujeres & 183 & 63.93 & 953 & \\
\hline \multirow[t]{2}{*}{ Distrito municipal } & Ciutat Vella & 242 & 62.81 & 894 & 0.8873 \\
\hline & Resto & 805 & 66.34 & 914 & \\
\hline \multirow[t]{2}{*}{ Grupo de riesgo } & UDVP & 770 & 65.06 & 982 & $<0.001$ \\
\hline & Homosexuales & 149 & 64.10 & 665 & \\
\hline \multirow[t]{2}{*}{$\mathrm{CD} 4$} & $14 \%$ & 273 & 72.16 & 629 & $<0.001$ \\
\hline & $>14 \%$ & 228 & 48.25 & 1396 & \\
\hline \multirow[t]{2}{*}{ Prueba tuberculina } & Positivo & 223 & 45.74 & 1914 & $<0.001$ \\
\hline & Negativo & 307 & 69.06 & 722 & \\
\hline \multirow[t]{2}{*}{ Sida } & Sí & 731 & 77.43 & 643 & $<0.001$ \\
\hline & No & 328 & 38.11 & 2549 & \\
\hline \multirow[t]{3}{*}{ Radiología } & Normal & 166 & 67.47 & 960 & 0.003 \\
\hline & Anormal cavit. & 146 & 52.05 & 1479 & \\
\hline & Anormal no cavit. & 697 & 67.29 & 835 & \\
\hline \multirow[t]{3}{*}{ Bacteriología } & Microscopía positiva & 402 & 63.93 & 951 & 0.025 \\
\hline & Sólo cultivo positivo & 342 & 69.88 & 833 & \\
\hline & Negativo & 188 & 57.98 & 948 & \\
\hline \multirow[t]{3}{*}{ Localización TBC } & Pulmonar & 503 & 58.25 & 1022 & $<0.001$ \\
\hline & Extrapulmonar & 362 & 72.10 & 833 & \\
\hline & Mixta & 184 & 71.74 & 867 & \\
\hline
\end{tabular}

* Mediana de supervivencia (se ha calculado en días).

En el ajuste multivariado de todas las variables, excepto CD4 y prueba de la tuberculina, se mostraron como predictores de la supervivencia las variables edad y sida (tabla 4, modelo A). No obstante, debido a la importancia de la variable CD4, puesto que el comportamiento de los valores no observados respecto a la supervivencia sugería que su distribución era aleatoria (figura 2), se propuso un modelo que no excluía esta variable (tabla 4, modelo B). En este caso, las variables sida, porcentaje de linfocitos CD4 y su interacción resultaron ser estadísticamente significativas, desapareciendo la influencia de la edad. Según este modelo, basado en la submuestra de 395 pacientes con información en todas las variables menos prueba de la tuberculina, el riesgo de morir en el período de seguimiento de un individuo con sida y nivel de CD4 inferior al $14 \%$ es 1,54 veces mayor que el riesgo de morir de un individuo con SIDA y nivel de CD4 superior al 14\%. Por otro lado, el riesgo de un individuo de morir con CD4 superior al $14 \%$ y de es 6,67 veces mayor que el riesgo de morir de un individuo con CD4 superior al $14 \%$ y sin sida.

Las figuras 3 y 4 muestran la interacción entre las variables sida y CD4, y en ellas pueden reconocerse cuatro patrones de supervivencia en función del nivel de CD4 y 
Tabla 4

Resultado de los modelos multivariados de Cox de los pacientes tuberculosos infectados por VIH en Barcelona entre 1988 y 1993. Modelo A: Incluyendo todas las variables excepto el porcentaje de CD4 y la prueba de la tuberculina. Modelo B: Incluyendo todas las variables excepto la prueba de la tuberculina

\begin{tabular}{|lccccc|}
\hline Modelo $A(n=805)$ & \multicolumn{1}{l}{} & & \\
\hline Variable & $\beta i$ & $D E^{*}$ & Valor $p$ & Riesgo & I.C. (95\%) \\
\hline Sida & 1.190 & 0.121 & $<0.001$ & 3.289 & $(2.597 ; 4.167)$ \\
Edad & 0.0292 & 0.006 & $<0.001$ & 1.030 & $(1.018 ; 1.041)$ \\
\hline Modelo B (n=395) & & & & & Riesgo \\
Variable & $\beta i$ & $D E^{*}$ & Valor $p$ & I.C. (95\%) \\
Sida & 0.3012 & 0.201 & 0.134 & 1.35 (CD4<14\%) & $(0.91 ; 2.00)$ \\
CD4 & & & & $(3.70 ; 11.11)$ \\
Sida * CD4 & 0.4365 & 0.153 & 0.004 & 1.54 (Sida) & $(1.15 ; 2.08)$ \\
\hline
\end{tabular}

Los niveles de referencia son no sida y porcentaje de CD4 superior al $14 \%$.

I.C. = Intervalo de confianza.

*DE $=$ desviación estándar.

Figura 3

Función de supervivencia para la variable «Linfocitos T CD4+» para pacientes con sida

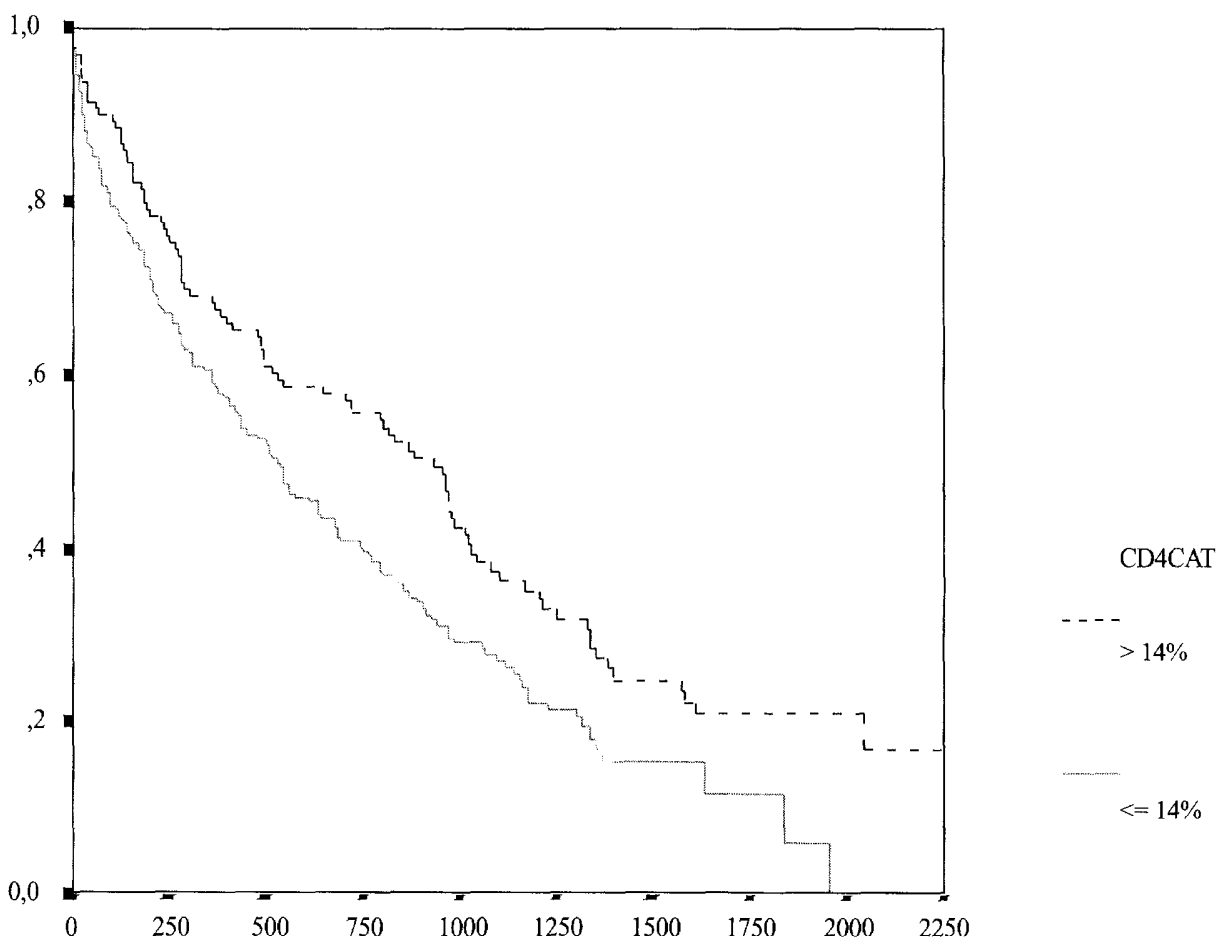

Días 
de la presencia o no de sida. En particular, puede observarse como el patrón de supervivencia de los pacientes menos inmunodeprimidos es siempre superior al de los más inmunodeprimidos. Dicho modelo ha sido validado mediante un análisis de los residuos de Cox-Snell (figura 5). Basándose en este modelo, la probabilidad de fallecer durante los primeros 9 meses después de haber iniciado el tratamiento antituberculoso, para un individuo con sida y con nivel de CD4 inferior al 14\% es del 32\%; para un individuo con sida pero con nivel de CD4 superior al $14 \%$ es del $22 \%$; para un individuo $\sin$ SIDA y nivel de CD4 inferior al $14 \%$ es del $25 \%$ y, finalmente, para un individuo sin sida y nivel de CD4 superior al $14 \%$ es del $4 \%$. Nótese como dichos valores se ajustan a los observados en las figuras 3 y 4 .

Figura 4

Función de supervivencia para la variable «Linfocitos T CD4+» para pacientes sin sida

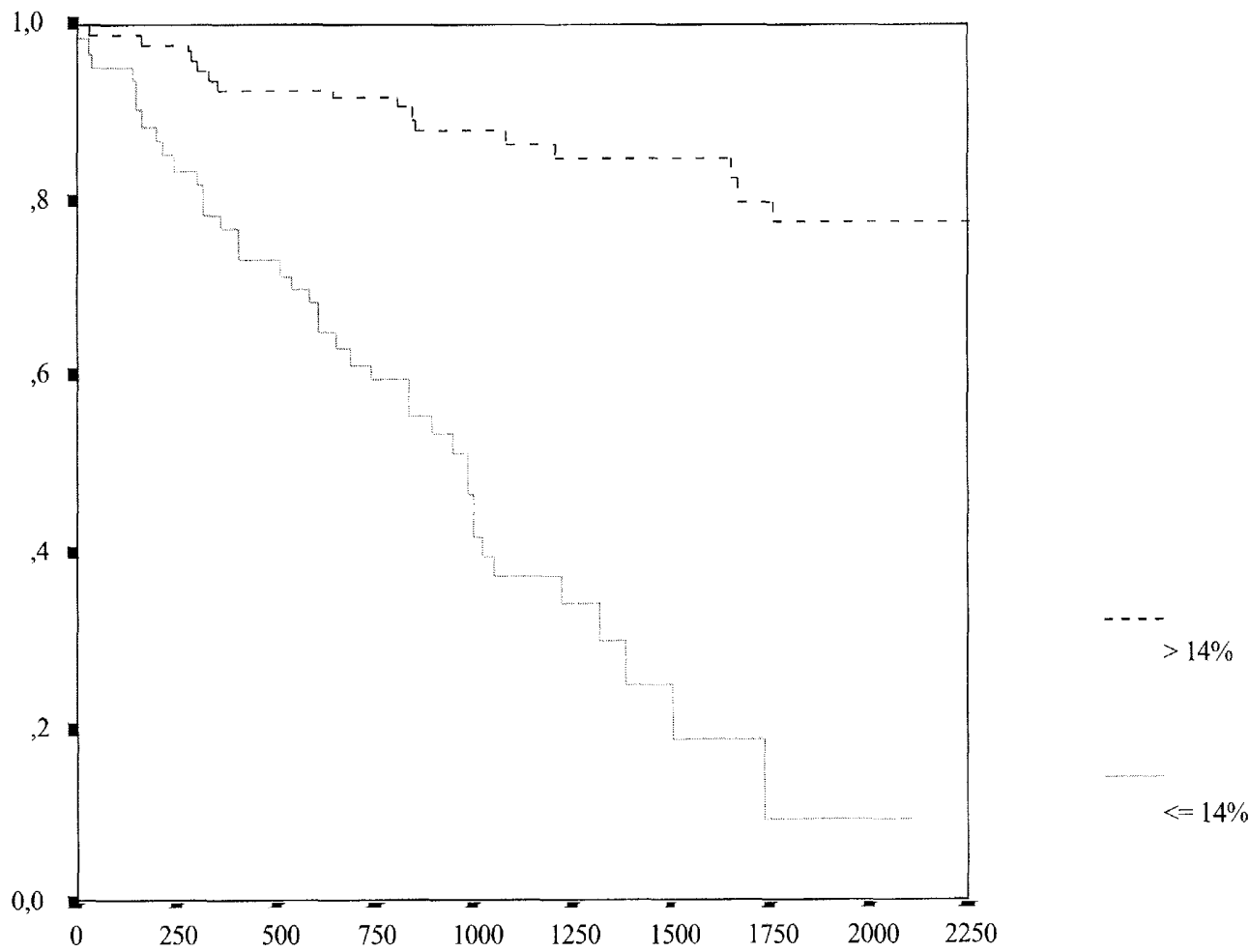

Días 
Figura 5

Residuos de Cox-Snell para el modelo multivariado de Cox excluyendo la variable «Prueba de la tuberculina»

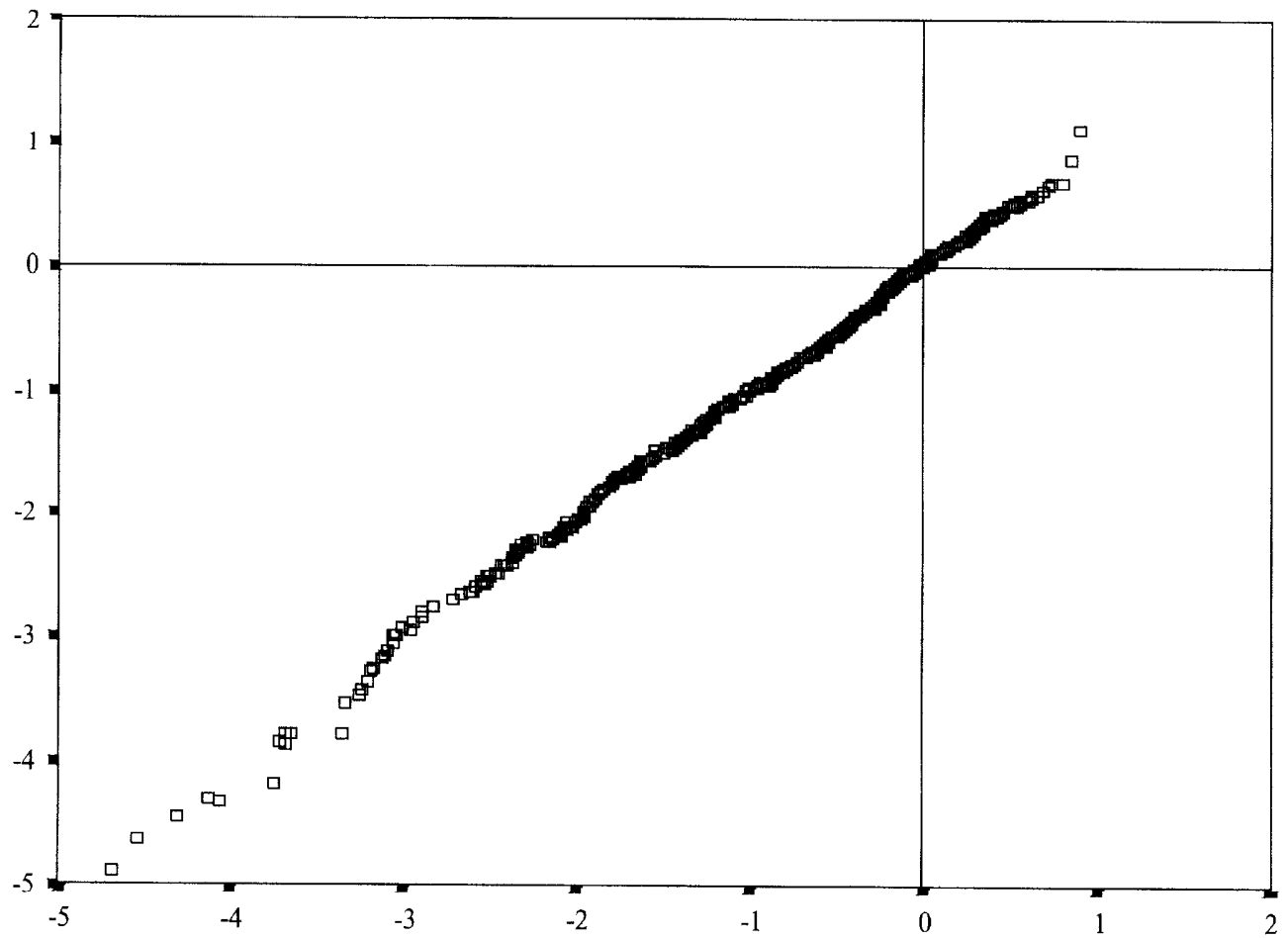

\section{DISCUSIÓN}

Si bien, los resultados del estudio se verán, probablemente, influidos por los nuevos tratamientos antirretrovirales, pensamos que no dejan de ser vigentes en los casos en que las sujetos no cumplan el tratamiento antirretroviral o bien lo rechacen, así como en la mayoría de los pacientes tuberculosos infectados por el VIH en los países donde dichos tratamientos no son accesibles.

Durante el período del estudio, el perfil de los pacientes infectados por el VIH ha cambiado en algunos aspectos. Así, la media de edad ha aumentado, hecho que puede ser debido al incremento anual en el porcentaje del grupo de hombres con prácticas homosexuales VIH positivos y diagnosticados con TBC, y a la disminución paralela en el porcentaje de UDVP, pues este colectivo suele ser, en nuestro medio, de menor edad que aquellos en que la vía de contagio ha sido la sexual ${ }^{14}$.

Por otro lado, es de destacar la elevada tasa de letalidad entre los pacientes VIH positivos con tuberculosis, ya que el $23 \%$ de ellos muere en los nueve primeros meses después de haber iniciado el tratamiento antituberculoso. No obstante, se ha observado que esta tasa varía desde un $4 \%$ en los pacientes tuberculosos VIH positivos sin diagnóstico de sida y con un recuento de linfocitos T CD4+ superior al $14 \%$, hasta el $32 \%$ observada en las personas tuberculosas VIH positivas diagnosticadas de sida y con un recuento de linfocitos $\mathrm{T}$ CD4+ inferior al 
$14 \%$. En cualquier caso, la tasa de letalidad de los pacientes tuberculosos VIH positivos es siempre superior a la tasa de un $3 \%$ observada en los tuberculosos VIH negati$\operatorname{vos}^{15}$.

En esta disminución han influido, probablemente, las actividades del Programa de Prevención y control de la TBC que se llevan a cabo en Barcelona desde principios de los noventa, especialmente en instituciones penitenciarias del distrito de Ciutat Vella, ya que se prioriza el tratamiento supervisado en los UDVP y, por lo tanto, podría explicar la disminución progresiva, a lo largo del período de estudio, del porcentaje de pacientes tuberculosos, seropositivos y UDVP.

En cuanto al estudio de supervivencia, se ha observado que los individuos que mueren durante los primeros nueve meses después de iniciar el tratamiento antituberculoso, se caracterizan por ser de edad más avanzada, presentar un porcentaje de linfocitos T CD4 inferior o igual a $14 \%$, tener un resultado negativo en la prueba de la tuberculina, no presentar antecedentes de prisión y haber sido diagnosticados de sida al inicio del tratamiento. Tanto la edad, como el porcentaje de linfocitos T CD4+, son variables que se han asociado frecuentemente a una peor supervivencia de los individuos infectados por el VIH ${ }^{16}$. Con relación a la prueba de la tuberculina, su valor predictor como factor de supervivencia en los pacientes tuberculosos también ha sido objetivado en diferentes estudios $^{17,18}$, así como el diagnóstico previo de sida ${ }^{19}$.

Llama la atención la mayor supervivencia de las personas que han estado en prisión en algún momento durante el período de estudio. Es probable que en ello influya la edad, ya que son pacientes más jóvenes, mayoritariamente UDVP y en los que, mientras están recluidos, el tratamiento antituberculoso suele estar más controlado ${ }^{20}$.

La variable localización anatómica de la TBC no se incluyó en el modelo multivaria- do de Cox, pues no cumple la hipótesis de riesgos proporcionales. Por otro lado, se puede observar una supervivencia mayor para los pacientes diagnosticados con TBC exclusivamente pulmonar a lo largo del tiempo; aunque en los nueve meses aún no se observa una diferencia significativa entre éstos y los diagnosticados con TBC extrapulmonar.

Diversos estudios han confirmado que tienen una supervivencia mayor las personas con TBC pulmonar que aquellas con TBC extrapulmonar o mixta ${ }^{7,21}$. También se ha objetivado que, en enfermos VIH positivos y diagnosticados de tuberculosis pulmonar, existe una probabilidad mayor de progresar a sida para aquellos individuos con edad más avanzada, patrón radiológico no cavitario, bajo cociente CD4/CD8 y resultado negativo en la prueba de la tuberculina ${ }^{22}$.

Los resultados presentados están en concordancia con diversos estudios llevados a cabo en otros países ${ }^{23,24}$ con respecto al CD4 y al sida, como variables de influencia en la supervivencia. Otros resultados indican que la TBC activa puede ser un marcador de inmunodepresión avanzada en pacientes VIH positivos, pudiendo acelerar el curso clínico de la infección por VIH.

Entre las limitaciones más importantes de este estudio cabe citar el elevado númcro de datos no observados en el porcentaje de linfocitos T CD4 $(53,6 \%)$ y en la prueba de la tuberculina $(49,8 \%)$. Para la primera variable dicho porcentaje va disminuyendo a lo largo del período de estudio debido, probablemente, a la creciente importancia que esta información ha ido tomando a lo largo de los años, como marcador de progresión en este tipo de pacientes. En la figura 2 , se observa cómo la curva de supervivencia correspondiente a aquellos pacientes en los que la variable CD4 no estaba informada, se sitúa entre las curvas de los pacientes con un porcentaje mayor y menor del $14 \%$. Esta distribución permite asumir la hipótesis de que estos datos esta- 
ban distribuidos al azar, lo que indicaría que la supervivencia de los pacientes con un valor no observado en el porcentaje de linfocitos T CD4 no es diferente a la de los pacientes con valores informados. En cambio, para los individuos con valor no observado en el resultado de la prueba de la tuberculina, cuya curva de supervivencia es similar a la de aquellos pacientes con un resultado negativo. Esto puede ser debido, probablemente, a que en los casos con diagnóstico clínico de TBC muy evidente, o aquellos casos en los que el enfermo presenta un estado de inmunodepresión muy severa, dicha prueba no se practica con la sistemática habitual. Así pues, la supervivencia de los pacientes con la prueba de la tuberculina informada no tiene porque ser representativa de la supervivencia de todos los individuos del estudio y, por este motivo, no se incluyó esta variable en el modelo de Cox.

Cuando en el modelo multivariado no se toman en consideración las variables CD4 y la prueba de la tuberculina, por presentar un alto porcentaje de datos desconocidos, resul$\tan$ significativas las asociaciones sida y edad, observándose una mejor supervivencia en aquellos individuos más jóvenes y sin diagnóstico de sida. Mientras que, cuando se analiza la submuestra de los 395 pacientes con todos los datos completos, las únicas variables que resultaron ser significativas en el modelo multivariado fueron sida, CD4 y su interacción, obscrvándose una mejor supervivencia para los pacientes sin sida y con CD4 superior al 14\%, desapareciendo la influencia de la edad.

En resumen, podemos concluir que la probabilidad de supervivencia en los pacientes con tuberculosis infectados por el VIH es muy variable, pero puede ser pronosticada en los países con pocos recursos económicos por variables como el diagnóstico previo de sida y la edad, y en los países desarrollados por el diagnóstico previo de sida, el recuento de linfocitos CD4 y la interacción SIDA-CD4.

\section{BIBLIOGRAFÍA}

1. Le point sur l'épidémie de SIDA: Décembre 1998. Onusida. Http://www.unaids.org/unaids/ document/epidemio.

2. Hirschel B, Francioli P. Progress and Problems in the fight againts AIDS. N Engl J Med 1998; 338: 906-908.

3. Palella FJ, Delaney K, Moorman A, Loveless M, Fuhrer J Satten G et al. Declining Morbidity and Mortality among Patients with advanced Human Immunodeficiency Virus Infection. N Engl J Med 1998; 338: 853-860.

4. Macroft A, Vella S, Benfield TL, Chiesi A, Miller V, Gargalianos $\mathrm{P}$, et al. Changing patterns on mortality across Furope in patients infected with HIV-1. Lancet 1998; 352: 1725-1730.

5. Macroft A, Johnson M, Phillips A. Factors affecting survival in patients with the acquired immunodeficiency syndrome. AIDS 1996; 10: 1057-1065.

6. García de Olalla P, Caylà JA, Brugal MT, Galdós H, Jansá JM, Clos R. Evolución de la mortalidad y supervivencia del SIDA en Barcelona (19811997). Med Clin (Barc) (en prensa).

7. Whalen C, Horsburg CR, Hom D, Lahart C, Simberkoff $\mathrm{M}$ and Ellner J. Accelerated Course of Human Immunodeficiency Virus Infection after Tuberculosis. Am J Crit Care Med 1995; 151: 129-135.

8. Centers for Disease Control. Revision of the CDC Surveillance Case Definition for Acquired Immunodeficiency Syndrome. MMWR 1987: 36 (Sup IS): 1S-15S.

9. Grupo de trabajo sobre tuberculosis. Consenso Nacional para el control de la Tuberculosis en España. Med Clin (Barc) 1992; 98: 24-31.

10. Collet D. Modelling Survival Data in Medical Research. Text in Statistical Sciences. London: Chapman \& Hall; 1994.

11. Norissis MJ. SPSS/PV + for yhe IBM PC/XT/AT. Chicago INC; 1986.

12. Epidemiological Graphics. Estimation and Testing package (EGRET). Seattle, Washington: Statistics and Epidemiology Research Corporation; 1990.

13. Borrell C, Plasència A, Pasarín M, Ortún V. Widening social inequalities in mortality: The case of Barceona, a Southern European City. J Epidemiol Community Health 1997; 51: 659-667. 
14. Brugal T, Caylà JA, Díez E, Galdós H, García de Olalla P, Jansà JM et al.Un pla de Prevenció i Control de la Sida per a Barcelona. Barcelona: Imprempta Municipal; 1998.

15. Chaisson RE, Clermont HC, Holt EA, Cantave M, Johnson MP and Atkinson J. Six-month supervised intermittent tuberculosis therapy in Haitian patients with and without HIV infection. Am J Respir Crit Care Med 1996; 26: 1259-60.

16. Mocroft A, Johnson MA, Phillips AN. Factors affecting survival in patients with the acquired immunodeiciency syndrome. AIDS 1996; 10: 1057-65.

17. Whalen C, Okwera A, Johnson J, Vjecha M, Hom $D$ and Wallis R. Predictors of Survival in human Immunodeficiency Virus-infected Patients with Pulmonary Tuberculosis. Am J Respir Crit Care Med 1996; 153: 1977-81.

18. Richter C, Koelemany, Swai BM, Perenboom R, Mwakyusa DH, Oosting J. Predictive markers of survival in HIV-seropositive and HIV-seronegative Tanzanian patients with extrapulmonary tuberculosis. Tuberc Lung Dis 1996; 76: 510-17.

19. Leroy V, Salmi LR, Dupon M, Sentilhes A, Teixer-Mangein J and Dequae L. Progression of $\mathrm{Hu}-$ man Immunodeficiency Virus Infection in $\mathrm{Pa}$ tients with tuberculosis Disease. Am J Epidemiol 1997; 145: 293.

20. Caylà JA, Marco A, Bedoya A, Guerrero R, García $J$ and Martin V. Differential characteristics of AIDS Patients with a History of Imprisonment. Int J Epidemiol, 1995; 24: 1188-1196.

21. Carcaba V, Cartón JA, Moris J, García Amorín Z, García Clemente $M$ and Rodríguez Junquera $M$. Tubcrculosis e infección por VIH. Evaluación de 132 casos. Rev Clin Esp 1993; 193: 12-16.

22. Caylà JA, Jansà, Artacoz L, Plasènsia A AIDS-TB Group. Predictors of AIDS in a cohort of HIV-infected patients with pulmonary or pleural tuberculosis. Tuberc Lung Dis 1993; 74: 113-120.

23. Selwyn PA, Alcabes P, Hartel D, et al. Clinical manifestations and predictors of disease preogression in drug users with human immunodeficiency virus infection. The $\mathrm{N}$ Engl J Med 1992; 327: $1697-1702$.

24. Alwood K, Keuly J, Moore-Rice, Stanton DL, Chailk CP, Chaisson RE, Effectiveness of supervised, intermittent therapy for tuberculosis in HIV-infected patients. AIDS 1994; 8: 1103-1108. 